\title{
Die Macht des Peripheren. Zu den Grenzen klarer Zuschreibungen anhand Winders Nachgeholten Freuden
}

\section{The power of the periphery. On the limits of unequivocal readings in Winder's Die nachgeholten Freuden}

Kristina Lahl

\begin{abstract}
While Ludwig Winder's novel Die nachgeholten Freuden (1927) appears at first glance to be a quite straightforward narrative of the interwar and post-Austro-Hungarian Bohemian region, it proves to be, at a second glance, bewilderingly enigmatic. Previously known certainties collapse within the storyline as central powers lose their influence and the periphery moves into the power vacuum - a plot line not unusual within interwar literature and in line e.g. with proletarian literature. Winder's unique technique, however, lies in the fact that many different interpretation approaches regarding e.g. a proletarian, a psychoanalytical or a religious reading, are being offered to the reader like on a silver platter but are disintegrating as soon as they are examined closely. This essay aims to reveal the discrepancies within the novel, but at the same time argues that these inconsistencies are deliberate and account for the mastery of the novel which thus articulates a complex commentary on the time and space of its origin.
\end{abstract}

\section{Keywords}

Bohemia; interwar; central powers; periphery; proletarian; psychoanalysis 


\section{1.}

Der Roman Die nachgeholten Freuden lässt sich geographisch wie historisch scheinbar eindeutig verorten: Der Ort des Geschehens, das Provinzstädtchen Boran, ist zwar fiktiv, verweist jedoch mit seinen geographischen Referenzen, z. B. auf die Stadt Brüx/Most, spezifisch auf Nordböhmen. Die Romanhandlung erstreckt sich vom Juli 1918 bis zum März 1926 und thematisiert damit die unmittelbare Nachkriegszeit und die erste Phase der Tschechoslowakischen Republik. Schon der allererste Satz des Romans beginnt mit einer genauen Datumsangabe: „Am 2. Juli 1918“1 - ebenso wie das letzte Kapitel des Romans: „Am 3. März 1926“.² Es ist davon auszugehen, dass Winder als Journalist, der das Tagesgeschehen genau beobachtete, diese Daten nicht gänzlich zufällig wählte. Der Beginn des Romans markiert auf den Tag genau die Anerkennung des Tschechoslowakischen Nationalrats als rechtmäßige provisorische Regierung der Tschechoslowakei durch die $\mathrm{USA}^{3}$ und das Ende des Romans die Woche, in welcher der in Locarno ausgehandelte Schiedsvertrag zwischen Österreich und der Tschechoslowakei im März 1926 unterzeichnet wurde. ${ }^{4}$ Gegeben ist somit ein Rahmen, der nicht nur die Zeit nach dem Ersten Weltkrieg in den Blick nimmt, sondern konkret auf die spezifische Situation in der Ersten Tschechoslowakischen Republik, mit dem besonderen Fokus auf der Loslösung von Österreich, gerichtet ist.

Es liegt somit nahe, diesen Roman Winders als einen tschechoslowakischen bzw. österreichischen zu rezipieren und seinen Autor ebenfalls in diesen historischen, geographischen und kulturellen Gesamtzusammenhang zu stellen. Nur: was genau bedeutet das? Welche Aussage wird damit getroffen und welchen Ansprüchen muss ein Text genügen, der als solcher kategorisiert wird ${ }^{5}$ Reicht die inhaltliche Thematisierung des historischen Bezugsrahmens aus, sind es eher stilistische Eigenheiten oder spezifische Schwerpunktsetzungen, welche die Literatur einer Region kennzeichnen, und inwiefern hat eine solche Einteilung überhaupt einen Mehrwert für die Literaturwissenschaft auBerhalb des verständlichen Wunsches nach Ordnung und Eindeutigkeit? Anhand von Winders Nachgeholten Freuden - das soll im Folgenden gezeigt werden -, lässt sich über diese Fragen trefflich diskutieren, weil er (auch dies ist möglicherweise ein Kriterium der regionalen Literatur) zahlreiche Referenzen aufweist und Analyseansätze einbezieht, sich jedoch letztendlich jeglicher Kategorisierung entzieht, die ihn eindeutig einem konkreten Interpretationszusammenhang zuordnen ließe. Vielmehr zeigt er eine Welt im

1 Winder, Ludwig (1987): Die nachgeholten Freuden [1927]. Wien - Hamburg: Paul Zsolnay, S. 7.

2 Winder [Anm. 1], S. 324.

3 vgl. Der Standard: Historischer Kalender. https://www.derstandard.at/story/1371170909437/historischer-kalender-2-juli (10. 08. 2020).

4 vgl. Koch, Klaus [u.a.] (hrsg.) (2002): Außenpolitische Dokumente der Republik Österreich 1918-1938, Band 5: Unter der Finanzkontrolle des Völkerbunds. Wien: Verlag für Geschichte und Politik, S. 493ff.

5 vgl. hierzu auch Krappmann, Jörg - Weinberg, Manfred (2014): Region - Provinz. Die deutsche Literatur Prags, Böhmens, Mährens und Sudetenschlesiens jenseits von Liblice. Mit Anmerkungen zu Franz Kafka als Autor einer Regionalliteratur. In: Becher, Peter [u.a.] (hrsg.): Prag - Provinz. Wechselwirkungen und Gegensätze in der deutschsprachigen Regionalliteratur Böhmens, Mährens und Sudetenschlesiens. Wuppertal: Arco, S. $17-52$. 
Zerfall, in der fest verankert geglaubte Macht- und Zugehörigkeitsverhältnisse dekonstruiert wurden. Machtzentren werden außer Kraft gesetzt und hinterlassen ein Vakuum, das von zuvor unterschätzten und marginalisierten Entitäten besetzt wird. Das System des Romans ist, ebenso wie dasjenige seines Protagonisten Dupic, ein System der Dezentralisierung und Dekonstruktion.

\title{
2.
}

Diese Dezentralisierung bezieht sich ganz offensichtlich, wenn auch bei Weitem nicht alleine, zunächst auf den geographischen Raum: Das Machtzentrum Wien, vor dem Ersten Weltkrieg Dreh- und Angelpunkt der Finanzwelt, der politischen Entscheidungen und des kaiserlichen Hofs, gerät plötzlich ins Abseits. ${ }^{6}$ Während zuvor all diejenigen, die nach Macht, Reichtum und Einfluss strebten, nach Wien pilgern mussten, verschiebt sich im Laufe des Romans das Machtzentrum zunehmend in die tschechoslowakische Provinz - und es sind nun die Wiener Architekten und Kaufmänner, die sich für einen lukrativen Auftrag oder einen Hinweis auf die Entwicklungen der Börse auf die Reise machen müssen und, wie in einer verkehrten Welt, in der zuvor von niemandem beachteten Kleinstadt Boran ihre Aufwartung machen. Dabei wird insbesondere die Hierarchieverschiebung zwischen dem zuvor übermächtigen Österreich und der unterschätzten Tschechoslowakei anhand der sich wandelnden finanziellen Lage deutlich, indem die tschechische Krone bald die entwertete österreichische Währung überflügelt hat:

\begin{abstract}
„[Dupic] kaufte an der Wiener Börse tschechoslowakische Industriepapiere und verkaufte sie an der Prager Börse. Er nützte den Kredit, der ihm in Wien eingeräumt wurde, ließ die österreichische Milliardenschuld wachsen - und erhielt die Aktien fast umsonst, weil die österreichische Krone von Tag zu Tag fiel.“7
\end{abstract}

Durch den Zerfall des Habsburgerreiches ist ein Machtvakuum entstanden, in dem alle zuvor geltenden Gesetzmäßigkeiten ausgehebelt sind. Die Karten werden neu gemischt, Kategorien von Zentrum und Peripherie scheinen zumindest für den Moment keine Gültigkeit mehr zu besitzen. Eng verbunden ist mit der räumlich-geographischen Dimension dabei selbstverständlich auch die soziale bzw. gesellschaftliche, ${ }^{8}$ indem auch hier die Machtzentren wechseln: Der ironische Unterton ist nicht zu überlesen, wenn es bei Ankunft der Delegation der Adeligen auf Schloss Boran 1918 heißt: „Um drei Uhr ertönte Hupen im Hof. Es hallte und heulte, als ob mehrere schlafende Hunde plötzlich angeschossen worden wären.“ Und im darauffolgenden Absatz: „Auch die Gräfin hatte

6 vgl. hierzu auch Pfoser, Alfred (2018): Eine Hauptstadt auf der Suche nach Stabilität. Zehn Bilder vom Wien 1918/1919. In: Austriaca 87. Finis Austriae. La chute de l'aigle bicéphale, S. 103-136.

7 Winder [Anm. 1], S. 248.

8 Zur umfassenden Verschränkung von Raum und Sozialem vgl. z. B.: Kessl, Fabian - Reutlinger, Christian (hrsg.) (2019): Handbuch Sozialraum. Grundlagen für den Sozial- und Bildungsbereich. Wiesbaden: Springer. 
die Signale gehört". ${ }^{9}$ Der Niedergang des Adels, der den Wechsel der Zeiten verschlafen hatte, und die Wachablösung durch das Volk (hier durch die Anspielung auf die Internationale verdeutlicht), werden auf den ersten Seiten des Romans somit relativ schonungslos offensichtlich.

Im Laufe des Romans wird der Adel vollständig in die Marginalität gedrängt, das letzte Festhalten an überkommener Etikette und verlorener Würde erscheint entweder grotesk-tragisch (Gräfin), melancholisch-hilflos (Graf) oder resignierend-selbstironisch (Allegra). Keine dieser Reaktionen erregt in irgendeiner Weise Mitleid oder Entgegenkommen seitens Dupic oder der neuen Herren im Schloss, der Bettler und Verbrecher. Das Zentrum ihrer Macht in Boran wird Stück für Stück verkleinert und zunehmend vom Peripheren der umliegenden Stadt und der peripheren Bevölkerungsteile gleichsam aufgefressen. ${ }^{10}$ Dies beginnt mit der Öffnung des Privatparks für alle Bewohner Borans, wird fortgesetzt mit der Besiedlung der Schlossräume durch Obdachlose und endet schließlich mit der Herabwürdigung der jüngeren Generation der Adeligen zu Bediensteten der Neureichen. Zugleich ersetzt Dupics neu errichtetes Gemeindehaus auch architektonisch den Adelssitz - das neue Zentrum Borans ist sowohl für alle zugänglich als auch zugleich ihr Untergang - es ist nun der Ort, zu dem alle streben und an dem sich alle versklaven.

An diesem Ort wird zugleich deutlich, dass auch die andere sozial mächtige Gruppierung, die das Potential besessen hatte, nach dem Krieg und dem damit einhergehenden Niedergang des Adels diesem vollständig den Rang abzulaufen, nämlich das Bürgertum, im Roman zunehmend vom Zentrum in die Peripherie gedrängt wird. ${ }^{11}$ Bei der, feierlichen Einweihung des Hauses lässt Dupic zuerst die Bürgerlichen Platz nehmen, damit sie bei Ankunft der Prostituierten und der Arbeiter nicht einfach verschwinden können, sondern sich der Konfrontation stellen müssen:

„,Mit den Bürgerlichen setzen wir uns nicht an einen Tisch', rief ein couragierter Achtzehnjähriger. - ,Gut, so vertreibt die Bürgerlichen, ihr seid ja die Mehrheit', lachte Dupic. ,Komm, Söhnchen, pack den Bürgermeister bei der Krawatte und sag ihm: ,Das ist mein Platz.“"12

Die Bürger des Städtchens Boran haben sich allesamt in Dupics Hände begeben, indem sie sich von ihm Geld liehen. Dupic macht keinen Unterschied zwischen Bürgern und Adeligen, Arbeitern und Verbrechern, allen leiht er Geld, von einigen verlangt er es zurück, andere erniedrigt er, wieder andere bleiben von ihm vollkommen unbehelligt. Die

9 Winder [Anm. 1], S. 9.

10 vgl. zur komplexen Situation des habsburgischen Hochadels in den einzelnen Regionen Österreich-Ungarns zwischen Zentrum und Peripherie am Ende des 19. und zu Beginn des 20. Jahrhunderts: Lalic, Daniel (2017): Der Hochadel Kroatien-Slawoniens. Zwischen Verlust, Verteidigung und Neuerwerb gesellschaftlicher Elitepositionen (1868-1918). Berlin - Boston: De Gruyter, S. 79ff.

11 vgl. zur allgemeinen Veränderung der Sozialstruktur nach 1918 auch Speier, Hans (2011): Die Angestellten vor dem Nationalsozialismus. Ein Beitrag zum Verständnis der deutschen Sozialstruktur 1918-1933. Göttingen: Vandenhoeck \& Ruprecht.

12 Winder [Anm. 1], S. 290. 
Willkür, mit der Dupic über alle Einwohner Borans herrscht, hebt das zuvor herrschende Machtgefüge aus den Angeln. ${ }^{13}$ Es ist ein beinahe gottähnliches Schicksalsprinzip, undurchschaubar und nicht vorhersagbar, mit dem Dupic alle Standesunterschiede und Zugehörigkeitsmerkmale nivelliert.

\section{3.}

Die Macht in Boran scheint nach 1918 nun allein in Dupics Händen zu liegen. Schaut man sich die Figur näher an, so könnte der Gegensatz zwischen den alten zentralen Mächten und Eliten und dem neuen Kräftezentrum nicht auffälliger sein. Dupic vereinigt in sich zahlreiche Merkmale des sozial Peripheren - er ist fremd, ärmlich gekleidet, ohne Etikette, dubioser Herkunft und von hässlicher Physiognomie:

„Insbesondere seine Manieren und sein Umgang mit der Zeit stehen im krassem programmatischen Kontrast zur Höflichkeit der Adelswelt und charakterisieren seine soziale Herkunft aus der bäuerlichen Welt, seinen gesellschaftlichen Aufstieg vom nicht-Ort des Landes." ${ }^{14}$

Gleichzeitig ist er zutiefst enigmatisch, unberechenbar und undurchschaubar. Sein Aussehen, sein Auftreten und seine Handlungen sind zutiefst widersprüchlich. Nur eines von vielen Beispielen soll hier dazu dienen, die Unmöglichkeit, Dupics Aussehen zu greifen und näher zu beschreiben, zu illustrieren: Der Lehrer, Elsas Vater, versucht sich dessen Gesicht zu vergegenwärtigen, aber ,jede Erinnerung war ausgelöscht, er wußte nur, einen dünnen langen Bart hat er, der Mund ist unsichtbar, er hat keinen Mund, er hat aber auch keine Augen, es ist unmöglich, sich Dupics Augen vorzustellen. "15 Auch in Bezug auf seine Taten entzieht sich Dupic jeglicher klaren Zuschreibung. Als Karl, Elsas Bruder und Kommunist, versucht, sich einen Reim auf Dupics Verhalten zu machen, muss er resigniert feststellen, „daß Dupic kein Wucherer, kein Geizhals war, sondern ein Mensch, der sich in keine Kategorie einreihen ließ. [...] Karl fühlte, was alle fühlten, die sich Dupics Dasein nicht erklären konnten." 16

In beinahe märchenhafter Weise gelingt es Dupic, sich den Dynamiken, welche die Zentrum-Peripherie-Dichotomie verstärken (können), zu widersetzen bzw. diese auszuhebeln: Es sind diese „kumulative[n] Effekte, welche die Dominanz des Zentrums über die Peripherie weiter vergrößern: Effekt der Dominanz, Effekt der Rückkoppelung, Informationseffekt, psychologischer Effekt, Modernisierungseffekt und

13 vgl. zu Willkür als Gegensatz zum Gerechtigkeitsprinzip auch Luhmann, Niklas (1995): Das Recht der Gesellschaft. Frankfurt a. M.: Suhrkamp.

14 Müller-Funk, Wolfgang (1999): Die grenzenlose Lust an der Macht. Ludwig Winders „Die nachgeholten Freuden“ als literarisches Lehrstück einer Theorie der Macht. Handlungskern und Indizien. In: Fiala-Fürst. Ingeborg (hrsg): Mährische Deutschsprachige Literatur. Eine Bestandsaufnahme. Olomouc, Univerzita Palackého, S. 129-147 (hier S. 131).

15 Winder [Anm. 1], S. 94.

16 Winder [Anm. 1], S. 152-153. 
Produktionseffekt." 17 Kurz zusammengefasst besagen diese Effekte, dass sich durch die Dynamiken der Zentrum-Peripherie-Dichotomie diese stetig verschärft, und es kaum Möglichkeiten gibt, aus dem Marginalen ins Zentrum zu rücken. Für Dupic jedoch gelten diese Gesetzmäßigkeiten nicht. Obwohl er theoretisch Attribute des Peripheren trägt - sowohl in Wien als auch in Boran ist er ein Fremder, er entstammt ärmlichen Verhältnissen, er verkehrt mit Verbrechern usw. - lässt er sich trotzdem keiner Minorität zuordnen, er entstammt nicht nur einem „nicht-Ort“, er besitzt geradezu eine nicht-Identität, was die normalerweise geltenden festen gesellschaftlichen Grenzen für ihn überschreitbar machen. Durch die Verlagerung seines Kapitals, seiner Produktionstätigkeiten und nicht zuletzt auch seiner Person und dem damit verbundenen Willen (der gott- oder teufelsähnlich ist) in die Peripherie, durch seine unnachgiebige Haltung gegenüber den zentralen Mächten und durch seine Vorliebe für das Marginalisierte und Groteske, verschiebt Dupic die Grenzen zwischen geographischem wie sozialem Zentrum und Peripherie und führt sie damit ad absurdum.

Das kleine Provinzstädtchen wird durch Dupics Ankunft in mehr als einer Hinsicht von der Peripherie ins Zentrum gerückt. Zum einen wird es plötzlich zu einem Ort, der, wie bereits erwähnt, auf einmal als ein Finanz- und Produktionszentrum gilt, das von hohen Politikern, Architekten, Facharbeitern und Wissenschaftlern besucht wird. Damit verschiebt sich auch die Außenwahrnehmung; auch von Wien oder Prag aus erscheint Boran nicht mehr peripher. Gleichzeitig wird das Groteske an dieser Entwicklung deutlich, als das Gemeindehaus in Boran gebaut wird und Dupic seinen Willen in Bezug auf die Gestaltung des Gebäudes durchsetzt: „Nur die Hoffnung, der monströse Bau in dem entlegenen Provinzstädtchen werde von unliebsamen Kritikern nicht entdeckt werden, ermutigte die Architekten, die Sünde zu begehen."18

Bedeutsamer ist aber der Umstand, dass auch die Eigenwahrnehmung der Bürger Borans sich wandelt - die Stadt wird durch Dupics Ankunft autark, indem Geld, Arbeit, Politik und Produktion vor Ort angesiedelt werden: Sie ist sich selbst das Zentrum: „Die Welt begann beim ersten Haus von Boran, ihr Ende war beim letzten Haus von Boran, was außerhalb dieser Welt lag, war unrettbar verloren, ob nah, ob fern, zu helfen war keinem, nicht den Gatten, nicht den Söhnen, schließen wir die Augen und füllen wir unsere Bäuche!"19 Für diese Unabhängigkeit von außen zahlen jedoch für die Bürger Borans einen hohen Preis, sie werden nämlich von Dupic absolut abhängig: „Die Beschränkung auf das Kleine ermöglicht die absolute Verfügbarkeit. Totalitarismus bedeutet zunächst: absolute Kontrolle über den Raum, Unmöglichkeit des Einzelnen, den markierten Herrschaftsraum zu verlassen." 20

Hierzu passt auch Dupics (schließlich nicht umgesetzter Plan) seine Fabriken mit einem menschenverachtenden Zusatz auszustatten. Der Fabrikraum im unteren und das Bordell im oberen Stockwerk sollten nur durch eine Glaswand getrennt sein, sodass alles

17 Havlíček, Tomáš [u.a.] (2008): Innere und äußere Peripherie am Beispiel Tschechiens. In: Mitteilungen der der Österreichischen Geographischen Gesellschaft 150, S. 299-316 (hier S. 302).

18 Winder [Anm. 1], S. 286.

19 Winder [Anm. 1], S. 91.

20 Müller-Funk [Anm. 14], S. 139. 
ständig beobachtet werden kann. Es ist dies ein dem Panopticon gegenteiliges Modell ${ }^{21}$ statt normgerechten Verhaltens und Selbstdisziplinierung wird durch die ständige Beobachtung der Sünde jegliche Form von Norm und Scham überwunden - auch hier dreht Dupics Prinzip die Machtstrukturen um und macht wiederum das Periphere zur Norm.

$\mathrm{Zu}$ seinen Lebzeiten ist Dupic in seiner Macht uneingeschränkt; die einzige, die ihm etwas entgegenzusetzen hat, ist Elsa, der es gelingt, durch Aktienspekulation genug Kapital anzuhäufen, um eine eigene Fabrik zu gründen. - Elsa macht übrigens ihr Geld hauptsächlich dank Poldihütten-Aktien, und einer der Hauptenergieversorger der historischen Poldihütte war der in Deutsch Kralupp gelegene Braunkohleschacht Elsa. ${ }^{22}$ Obwohl auch Elsas Einfluss gering bleibt, ist sie dennoch die einzige Figur, der es im Laufe des Romans gelingt, ihren eigenen Weg zu gehen, ihre Würde zu bewahren, ihre Ziele umzusetzen und schließlich etwas Glück zu erlangen. Dabei ist auffällig, dass sie als Jüdin und als Frau eine Figur an der sozialen Peripherie ist. Es ist auch kein Zufall, dass ausgerechnet ein Kind schließlich die Macht Dupics bricht, und dass diejenigen, die schließlich sein Erbe antreten, die Bettler und Verbrecher sind, die er im Schloss einquartiert hat. Er schafft einen Herrschaftsraum in Boran, in dem die neuen Mächte nicht die erwartbaren und etablierten Hierarchien festigen, sondern gerade im Gegenteil das Marginalisierte zum Handlungsträger machen: „Das Fluchen der Männer und Frauen berührte ihn nicht. Kein Mann und kein Weib war ihm gewachsen. Es war ein Kind, das ihn zu Fall brachte."23

\section{4.}

Das Verhalten der Einwohner des Provinzstädtchens, das durch einen unerwartet verlaufenden Besuch zu Reichtum und Macht, aber auch zu totalitären Verhältnissen kommt, erinnert an das fast 30 Jahre später entstandene Drama Der Besuch der alten Dame von Friedrich Dürrenmatt. Es gibt überraschend analoge Textstellen ${ }^{24}$ und Ähnlichkeiten im Umgang mit dem Grotesken. ${ }^{25}$ Im Vergleich zwischen diesen beiden Werken kann man beispielhaft das herausarbeiten, was den Roman Winders auch im Gegensatz zu zahlreichen anderen Texten auszeichnet: Bei Dürrenmatt lässt sich nämlich die zentrale Frage, die Frage nach der Schuld, zwar nicht endgültig klären, aber man kann sie zumindest stellen und diskutieren. Es gibt unterschiedliche Interpretationen, die jeweils ihre Berechtigung besitzen und in denen eine oder mehrere mögliche Antworten liegen, deren Zusammenschau ein Gesamtbild ergibt. Bei Winder erscheint es jedoch müßig, eine solche Frage - etwa in Bezug auf die Motivation des Protagonisten oder in Bezug auf den Sinn und die Dynamiken des Geschehens - überhaupt zu stellen; denn selbst

\footnotetext{
21 vgl. Bentham, Jeremy (2009): Panopticon. Or the Inspection House [1791]. Whitefish: Kessinger.

22 vgl. Archiv Komotau: http://alte-hp.komotauarchiv.de/poldihuette.htm (12. 08. 2020).

23 Winder [Anm. 1], S. 324

24 vgl. die Beschreibungen, in denen sich die zuvor verarmten Bürger von Boran und Güllen wieder mit Lebensmitteln, Kleidung und schließlich Luxusgütern ausstatten.

25 So z. B. in der Darstellung der Körperlichkeit der ProtagonistInnen.
} 
wenn man sie stellt, finden sich zwar Interpretationsansätze, diese erweisen sich aber allesamt als Finten:

Es liegt nahe, die Figur des Adam Dupic mithilfe psychoanalytischer Ansätze zu interpretieren. Dafür spricht die ausführliche Beschreibung seiner Jugendjahre, in denen er, auf engstem Raum mit seiner Familie in einer Bauernstube hausend, die sexuellen Exzesse seiner Mutter und ihrer Magd miterleben muss, was bei ihm eine lebenslange, unheilvolle Vermischung von Ekel und Begierde hervorruft. ${ }^{26}$ Dafür spricht auch seine spätere Rückkehr, in der er seinen Vater rächt, und seine Fixierung auf das Haus seiner Kindheit und das Bett seiner Mutter, das er fortwährend als Mobiliar mit sich umziehen lässt.

Und doch erweist sich dieser Deutungsansatz, verfolgt man ihn mit der nötigen Konsequenz, als unbefriedigend: „Aber irgend etwas stimmt da nicht, ganz einfach scheint der Fall nicht zu liegen." ${ }^{27}$ Das gesamte Kapitel 5, in dem Dupics Herkunft geschildert wird, wirkt märchenhaft (Wanderschaft, Lehrjahre, kometenhafter Aufstieg, triumphale Rückkehr), teilweise unglaubwürdig und insgesamt äußerst seltsam - einzelne Verhaltensweisen und Motivationen werden nicht erläutert und bleiben im Dunkeln: Die Ablehnung der Bauern im Dorf, der Verfolgungswahn Dupics und auch seine nachfolgenden Machtphantasien ergeben sich nicht zwangsläufig aus den im Roman geschilderten Jugenderlebnissen. Fraglos ist die psychoanalytische Komponente, die Müller-Funk auch in der Erklärung des enigmatischen Romantitels herausarbeitet, ${ }^{28}$ vorhanden, allerdings wirft sie mehr Fragen auf, als dass sie diese beantwortet. Der Versuch, die Figur Adam Dupic aus seiner Herkunft her zu deuten, muss zwangsläufig scheitern.

Die Ungereimtheiten setzen sich auch in Peter Dupics Versuch, das Verhalten seines Vaters psychologisch zu deuten, fort. Obwohl er als Arzt eine gewisse Einsicht in psychoanalytische Vorgänge besitzt, liegt er wiederholt falsch, wenn er prophezeit, wie und mit welchen Beweggründen sich sein Vater verhalten wird. Auch seine Deutung des Vaters als des Urbösen, das sich ihm im Krieg offenbart, stößt an offensichtliche Grenzen:

\begin{abstract}
„Dann aber, im Krieg, hab“ ich immer öfter an dich denken müssen. Alles, was geschah, war mir seltsam vertraut. Das Morden. Das sinnlose Kämpfen um jeden Fußbreit Boden. Die mörderischen Empfindungen, die Giftgase, die Tanks. Da war wieder das Böse. Die Urkraft. Als ob alles von Dir ausginge. Als ob Du plötzlich zur Macht gelangt wärst. Einen schönen Tag hatte ich im Krieg, das war der Tag, an dem ich erfuhr, daß du deinen Besitz aufgabst.“29
\end{abstract}

Vor der Aufgabe seines Besitzes war Vater Dupic jedoch ein weitestgehend unbescholtener Bauer. Er pflegte zwar eine Feindschaft mit seinen fünf Nachbarn, dies jedoch nicht durch Morden und Brandschatzen:

26 vgl. auch analoge Stellen in Herman Ungars Die Verstümmelten.

27 Winder [Anm. 1], S. 147.

28 vgl. Müller-Funk [Anm. 14], S. 132.

29 Winder [Anm. 1], S. 164. 
„Es gelang ihm fast unmerklich, an die Besitzungen der Bauern heranzurücken, denen er konsequent ein freundliches Gesicht zeigte. Der reichste Mann war der devoteste. Sein Leben war friedliche Arbeit, ereignislose Gleichförmigkeit. Die Jahre vergingen wie ein heißer Arbeitstag. “ 30

Entweder der Erzähler ist höchst unzuverlässig - oder aber Peter Dupics Erinnerungen sind es. Seine ,Mission“, den Vater vor sich selbst zu retten, an der er schließlich scheitert, ergibt sich aus nicht eindeutig motivierten und dadurch bis zu gewissen Graden nicht nachvollziehbaren Beweggründen.

Diese Problematik der unzureichenden psychologischen Deutung der Figuren und ihrer Handlungen hat ihren hauptsächlichen Grund in der Vermischung mit einem anderen Aspekt, der als Bedeutungsebene unmittelbar in den Blick fällt und auch mit der Figur des Sohns eng verbunden ist: dem Religiösen. ${ }^{31}$ An mehreren Stellen des Romans wird Adam Dupic mit teuflischen Attributen gezeichnet - und Peter Dupic bezeichnet seinen Vater an einer Stelle direkt als Antichristen: „Vor neunzehnhundertneunzehn Jahren ist Jesus Christus ausersehen worden, alle guten Kräfte, deren die Menschheit fähig ist, in sich zu vereinigen. Vor etwa sechzig Jahren ist mein Vater ausersehen worden, ein Krater aller bösen Kräfte der Menschheit zu werden. " ${ }^{22}$ Eine äußerst interessante Parallele findet sich zu einem anderen Text aus dem späten 20. Jahrhundert, nämlich Stephen Kings Needful Things. Auch hier gelangt ein Fremder, der relativ offensichtlich mit dem Teufel gleichgesetzt werden kann, in eine Kleinstadt und versklavt deren Bürger durch zunächst als Wohltat erscheinende Gaben. ${ }^{33}$ Die Geschichte und damit auch die Aussage entwickeln sich bei Winder und King allerdings schließlich unterschiedlich - dies liegt an der unterschiedlichen Perspektive: King richtet den Fokus auf die Stadt und ihre Bürger, und deswegen ist die Motivation Gaunts im Endeffekt unerheblich der Lesende akzeptiert schlicht und einfach, dass dieser das Böse will. Bei Winder liegt der Fall nicht so einfach, er ist um einiges komplexer und unauflöslicher: Im Fokus der Erzählung steht Dupic selbst, und seine Motivation gibt Rätsel auf; gegensätzliche Deutungsansätze finden sich zum einen indirekt in der Vorgeschichte Dupics, die eine psychoanalytische Motivation nahezulegen scheint, dann in der direkten Deutung des Sohns, der den Vater als Ausgeburt des Bösen ansieht, vor dem es die Stadt und auch ihn selbst zu retten gilt; und schließlich in den Selbstaussagen Dupics, der sich, wenn auch teils ironisch, durchaus als Wohltäter und gottähnlichen Schöpfer stilisiert. Die einzige Figur, die ein kohärentes religiöses (in diesem Fall gnostisches) Weltbild zu haben scheint, Peter Dupic, muss sich im Verlauf des Romans wiederholt eingestehen, dass er sich in der Interpretation und Vorhersage des Verhaltens seines Vaters täuscht. Er ist zwar eine positiv konnotierte, aber zum Schluss farb- und belanglose Figur. Wenn

30 Winder [Anm. 1], S. 67.

31 Religion spielt in Winders Werken wiederholt eine zentrale Bedeutung - am offensichtlichsten ist dies in seinem Frühwerk Die jüdische Orgel.

32 Winder [Anm. 1], S. 176.

33 Dupic kann es sich dabei leisten, in seinen Gaben sehr viel fantasieloser als Kings Protagonist, Leland Gaunt, zu sein, da nach dem Ersten Weltkrieg nichts „more needful“ ist als das Notwendigste zum Überleben. 
überhaupt eine religiöse Deutung im Roman vorgenommen werden kann, so beschränkt sie sich auf die realistische, aber recht wenig zufriedenstellende Einsicht, dass über Dupics Handeln und Gottes Wollen keine rationale Aussage getroffen werden kann, wie diese in sich zutiefst widersprüchliche Stelle zeigt:

„Gab es aber einen Sinn der Schöpfung, den [Dupic] nicht kannte, eine göttliche Weltordnung - : so wollte er hier, in Boran, in diesem kleinen Kreis, inmitten dieser gleichgültigen Menschen, einbrechen in diese Ordnung und eine eigene Ordnung schaffen, eine göttlich unvernünftige, göttlich chaotische Ordnung, erkennbar keinem außer ihm selbst. “"

Eine andere Möglichkeit, den Roman zu interpretieren, scheint die antikapitalistische Lesart zu sein. Dupic erscheint über Phasen des Romans als teuflische Ausgeburt der Finanzwelt, nur durch sein am Aktienmarkt skrupellos erworbenes Kapital ist es ihm möglich, sein ausbeuterisches System aufzubauen. Aus diesem Grunde überrascht es nicht, dass er kommunistische Figuren gegen sich aufbringt - so z. B. Elsas Bruder Karl und den Arbeiter Domansky. Doch obwohl Dupic zu Beginn offensichtlich die Feindfigur ist, hat Karl schon bald Probleme, Dupic und sein System zu greifen: „Karl forschte die Bevölkerung aus. Das Bild, das er gewann, war sehr verwirrend. Je mehr er erfuhr, desto rätselhafter wurde der Mann Dupic. Ein System schien Dupic nicht zu haben, Widersprüche häuften sich. “35 Die Kommunisten im Roman scheitern in ihrem Versuch, Dupic etwas entgegenzusetzen, da sie in ihm kein kohärentes Feindbild ausmachen können, sondern er sich ihnen durch seine Ambivalenz ständig entwindet. Die sozialkritischste Perspektive äußert erstaunlicherweise eine andere ambivalente Nebenfigur, nämlich die weitsichtige und dennoch im Kindlich-Staunenden und Passiven verbleibende junge Gräfin Allegra, die in ihren Phantasien das System Dupic zu einem grotesken, aber durchaus folgerichtigen Ende denkt:

„Sie phantasierte, eines Tages werde Dupic der Mama zwanzig Millionen unter der Bedingung schenken, daß sie ihn täglich zum Dejeuner lade [...]; eines Tages würde Dupic ein gebratenes Bauernmädchen servieren lassen und Mama zwingen, einen Schenkel mit Mayonnaise zu verspeisen. ${ }^{\text {"36 }}$

Doch dazu kommt es schließlich nicht, nicht zuletzt auch deshalb, weil ein solches Verhalten Dupic mit einem deutlichen antikapitalistischen und anti-aristokratischen Anstrich versehen würde, der in dieser Eindeutigkeit eben nicht vorhanden ist. Allegra prophezeit zu Beginn des Romans einen Aufstand des Volkes gegen den Adel im Sinne des Klassenkampfes:

34 Winder [Anm. 1], S. 79.

35 Winder [Anm. 1], S. 151.

36 Winder [Anm. 1], S. 120. 
„Und da werden wir alle daliegen wie Wasserleichen, und über uns wird ein riesiger, unerhört gewalttätiger Elefant mit schrecklichen, wilden Augen stehen, und wir werden wissen: Das ist das Volk! [... U]nd wir, wir werden nix sehn, wir werden nur fühlen: Jetzt ist er über uns, und wenn er nur ein bissel auszuschlagen anfangt, sind wir alle hin. [...] Weil wir doch immer geglaubt haben, der Elefant ist nur zu unserem Vergnügen da, und je größer und geduldiger er ist, desto mehr dürfen wir ihm aufladen.“37

Jedoch muss sie später feststellen, dass das System, das die alten zentralen Eliten abgelöst hat, nicht das Volk ist, sondern Dupic, der sich nicht einordnen lässt: „Aber nein, [...] ich hab، das Volk gemeint, das Volk, verstehst, wird auf uns herumtrampeln, das hab“ ich gemeint. Gehört der Dupic zum Volk? Zu uns gehört er auch nicht, was ist's also mit ihm, wohin gehört er?"38 Aus dem Kampf der Mächte gehen nicht das Volk oder der Sozialismus erfolgreich hervor, sondern eher Willkür und Chaos. Eine antikapitalistische Lesart des Romans hält somit nicht stand - es wird eher ein desolates Bild der Arbeiterbewegung gezeichnet:

\begin{abstract}
„Da hat es jahrzehntelang eine erfolgreiche Arbeiterbewegung gegeben, da ist jahrzehntelang ein sogenanntes Klassenbewußtsein in der Arbeiterschaft geweckt worden, in mehreren Staaten sind die Arbeiter sogar zur Regierung gelangt - und ein einziger Mensch bringt es mit Leichtigkeit fertig, alle Gesetze der Zeit umzustoßen. “39
\end{abstract}

Auch Deutungsansätze in Richtung von Stellungnahmen zum Nationalitätenstreit wären denkbar und lohnenswert in einem Roman, der im Jahre 1927 in der Tschechoslowakei erschien und ganz konkret Ereignisse zum Thema hat, die in direktem Bezug zum Niedergang des Habsburgerreiches und zum Entstehen des neuen Staates stehen. ${ }^{40}$ Und doch berühren diese historischen Bezugspunkte das Geschehen in Boran nur peripher - das System Dupic scheint zwar eine Ausgeburt seiner Zeit zu sein, allerdings bleibt es diffus, inwiefern die politischen Umstürze tatsächlich mit Dupic zusammenhängen. Dupic scheint die Entwicklungen und Absichten der einzelnen nationalen und politischen Parteien jeweils genau vorhersehen zu können, sie tangieren ihn jedoch nicht, weder im positiven noch im negativen Sinne. Für ihn sind die Aktionen aller Seiten gleich lächerlich. So werden z. B. die Schicksalstage vom Oktober 1918 abgewertet:

„Die gräfliche Familie blieb unsichtbar, die Fenster im Schloß waren verhängt, in der Wohnung des Rechtsanwalts Dr. Podlesny in der Palackystraße wurde die Revolution vorbereitet, die Deutschen kämpften noch immer für Kaiser und Reich. Dupic lächelte, es war ein Spaß, ein herrliches Amüsement.“41

Winder [Anm. 1], S. 35-36.

40 Wie auch zahlreiche andere Romane der Zeit und der Region - z. B. F.C. Weiskopfs Das Slawenlied.

41 Winder [Anm. 1], S. 101-102. 
Die bisher geäußerten Zweifel ob der Verortung von Winders Roman in eindeutige Kategorien erstrecken sich nicht zuletzt auch auf die rein räumliche Komponente und auf den Chronotopos des Textes, obwohl Winder sehr genaue zeitliche und recht präzise geographische Angaben macht. Das Changieren zwischen einer realhistorischen Verortung einerseits und einer mit magischen und märchenhaften Elementen gespickten Handlung andererseits, hat Müller-Funk herausgearbeitet:

\begin{abstract}
„[Die Erzählung] operiert realistisch, indem sie den Leser auf historische Ereignisse verweist und geschichtliche Namen des böhmischen Adels zitiert oder den Nationalitätenkampf zwischen Tschechen und Deutschen erwähnt. [...] Die religiöse Deutung hingegen avisiert einen Leser, der die Handlung wie in einem Märchen nachvollzieht. [...] So verklammert Winder zwei Textsorten, zwei literarische ,Sprachspiele‘ in einem Roman. Einerseits verwendet Winder Momente märchenhafter Verfremdung und rückt seinen Roman in die Nähe der Surrealität von Borges und dessen literarische Spekulation über mögliche Welten. Andererseits implementiert er Zeitbezüge, die eine ,realistische‘ Lektüre nahelegen. Dabei kann sich der Leser nicht für eine der beiden Lesarten entscheiden, sondern muß beständig die eine durch die andere überblenden." ${ }^{42}$
\end{abstract}

\title{
5.
}

Die Antwort auf die Frage des Tagungsbandes in Hinblick auf die tschechoslowakische und/oder österreichische Dimension in Winders Schaffen kann dementsprechend, zumindest in Bezug auf den Roman Die nachgeholten Freuden, immer nur eingeschränkt mit einem ,ja, aber... ausfallen. Die einzelnen Lesarten bleiben jeweils für sich allein genommen unbefriedigend, indem sie unglaubhaft, widersprüchlich und inkonsequent erscheinen. Gemeinsam und in Summe ergeben sie jedoch ein Gesamtbild des Romans, der sich die Dezentralisierung, in diesem Falle die Aushebelung fester Normierungen, zum Prinzip macht und damit den Versuch eindeutiger Antworten und klarer Zuschreibungen ad absurdum führt.

Heck u. a. ${ }^{43}$ arbeiten heraus, inwiefern die Zentrum/Peripherie-Dichotomien ein strukturgebendes Merkmal von Kafkas Texten sind: Als Beispiel nennen sie etwa die Werke Das Schloss, Der Prozeß und Vor dem Gesetz, wo jeweils der periphere Protagonist auf eine zentrale Macht stößt, ins Zentrum jedoch nie vorstoßen kann - die Differenz zwischen Zentrum und Peripherie, die den Hauptkonflikt der Texte ausmache, und das damit einhergehende Machtgefälle äußere sich insbesondere in der vollkommenen Aussichtslosigkeit des peripheren Protagonisten, das Wesen des Zentrums zu greifen oder gar zu erobern. Es ist von Rezensenten des Öfteren bemerkt worden, dass Win-

\footnotetext{
42 Müller-Funk [Anm. 14], S. 138.

43 vgl. Heck, Justus [u.a.] (2011): Ideelle Zentren und Peripherien. In: Arbeitsgruppe „Zentrum und Peripherie in soziologischen Differenzierungstheorien" (hrsg.): Mythos Mitte. Wirkmächtigkeit, Potenzial und Grenze der Unterscheidung ,Zentrum/Peripherie‘. Wiesbaden: Springer, S. 69-104.
} 
ders Roman Nachgeholte Freuden ein Gegenstück zu Kafkas Schloss ${ }^{44}$ sei - und im Sinne der Umkehrung der Zentrum-Peripherie-Dichotomie ist er tatsächlich als ein solches zu lesen; das Zentrum ist für die Peripherie nicht nur erreichbar, sondern es wird vollkommen erobert, die Verhältnisse werden von Grund auf umgekehrt - das Marginale rückt ins Zentrum, das ehemals Zentrale wird an den Rand gedrängt und marginalisiert. Doch auch wenn die Romane von Kafka und Winder in dieser Hinsicht entgegengesetzte Voraussetzungen haben, so haben sie doch im Kern den gleichen Ansatz: Sowohl durch die undurchschaubare Übermacht des Zentrums bei Kafka, als auch durch die Entmachtung und Bloßlegung der zentralen Strukturen bei Winder wird Bedeutung dezentralisiert und zerfasert: Das enigmatische, respektive das zerschlagene Machtsystem gibt keine eindeutigen und sinnstiftenden Antworten; Lesende können sich in hermeneutischen Fingerübungen schulen - doch end- und allgemeingültige Aussagen zur Verfasstheit der modernen Welt und der Rolle des Individuums in ihr werden ihnen nicht geboten.

Man könnte nun dieses Prinzip wiederum zum Merkmal deutschböhmischer und deutschmährischer Literatur erküren, indem sich ja auch die Texte von Franz Kafka und z. B. Hermann Ungar als Meisterwerke des ,ja, aber...' erweisen. Wie sinnvoll dies jedoch ist, bleibt offen. Denn offensichtlich liegt in der Synthese vermeintlich unvereinbarer Ambivalenzen und im bewussten Verzicht auf eindeutige Grenzziehungen der literarische Genuss und die stimulierende geistige Bereicherung bei der Lektüre Winders.

\section{Quellen und Literatur}

Bentham, Jeremy (2009): Panopticon. Or the Inspection House [1791]. Whitefish: Kessinger.

Havlíček, Tomáš u.a.] (2008): Innere und äußere Peripherie am Beispiel Tschechiens. In: Mitteilungen der Österreichischen Geographischen Gesellschaft 150, S. 299-316.

Heck, Justus [u.a.] (2011): Ideelle Zentren und Peripherien. In: Arbeitsgruppe „Zentrum und Peripherie in soziologischen Differenzierungstheorien“ (hrsg.): Mythos Mitte. Wirkmächtigkeit, Potenzial und Grenze der Unterscheidung ,Zentrum/Peripherie‘. Wiesbaden: Springer, S. 69-104.

Kessl, Fabian - Reutlinger, Christian (hrsg.) (2019): Handbuch Sozialraum. Grundlagen für den Sozial- und Bildungsbereich. Wiesbaden: Springer.

Koch, Klaus [u.a.] (hrsg.) (2002): Außenpolitische Dokumente der Republik Österreich 1918-1938, Band 5: Unter der Finanzkontrolle des Völkerbunds. Wien: Verlag für Geschichte und Politik.

Krappmann, Jörg - Weinberg, Manfred (2014): Region - Provinz. Die deutsche Literatur Prags, Böhmens, Mährens und Sudetenschlesiens jenseits von Liblice. Mit Anmerkungen zu Franz Kafka als Autor einer Regionalliteratur. In: Becher, Peter [u.a.] (hrsg.): Prag - Provinz. Wechselwirkungen und Gegensätze in der deutschsprachigen Regionalliteratur Böhmens, Mährens und Sudetenschlesiens. Wuppertal: Arco, S. 17-52.

Lalic, Daniel (2017): Der Hochadel Kroatien-Slawoniens. Zwischen Verlust, Verteidigung und Neuerwerb gesellschaftlicher Elitepositionen (1868-1918). Berlin - Boston: De Gruyter.

Luhmann, Niklas (1995): Das Recht der Gesellschaft. Frankfurt a. M.: Suhrkamp.

44 vgl. Winder [Anm. 1], Klappentext. 
Müller-Funk, Wolfgang (1999): Die grenzenlose Lust an der Macht. Ludwig Winders „Die nachgeholten Freuden“ als literarisches Lehrstück einer Theorie der Macht. Handlungskern und Indizien. In: Fiala-Fürst, Ingeborg (hrsg.): Mährische deutschsprachige Literatur. Eine Bestandsaufnahme. Olomouc: Univerzita Palackého, S. 129-147.

Pfoser, Alfred (2018): Eine Hauptstadt auf der Suche nach Stabilität. Zehn Bilder vom Wien 1918/1919. In: Austriaca 87. Finis Austriae. La chute de l'aigle bicéphale, S. 103-136.

Speier, Hans (2011): Die Angestellten vor dem Nationalsozialismus. Ein Beitrag zum Verständnis der deutschen Sozialstruktur 1918-1933. Göttingen: Vandenhoeck \& Ruprecht.

Winder, Ludwig (1987): Die nachgeholten Freuden [1927]. Wien - Hamburg: Paul Zsolnay.

Dr. phil. Kristina Lahl / kristina.lahl@kit.edu

KIT - Karlsruher Institut für Technologie, DE Forschungsförderung (FOR), Campus Süd, FritzErler-Str. 1-3, 76133 Karlsruhe, Deutschland 